\title{
Lois de croissance en biomasse du taillis : le robinier dans le Val-de-Loire
}

\author{
L. PAGES * \\ INRA, Centre de Recherches dOrléans \\ Station de Sylviculture, Ardon, F 45160 Olivet
}

\section{Résumé}

L'évolution de la masse ligneuse totale sur pied (ou production utile) est étudiée dans le cas des taillis traditionnels de robinier (Robinia pseudacacia L.) en Val-de-Loire.

Des tarifs «biomasse brin» sont établis sur un échantillon de 217 individus. Les relations sont du type $: b=a_{n}+a_{1} \cdot c^{\alpha} . H^{\beta}$ pour les brins de plus de $1,30 \mathrm{~m}$ de hauteur (b est la masse ligneuse totale du brin, c sa circonférence à $1,30 \mathrm{~m}$, HD la hauteur dominante du peuplement d'appartenance); et $: b=a_{1}+a_{1}$, h* pour les brins de moins de $1,30 \mathrm{~m}$ (h est la hauteur du brin).

Ces tarifs, appliqués aux inventaires de 31 placettes échantillons, âgées de 1 à 25 ans, permettent de reconstituer l'évolution de la masse ligneuse sur pied. Un modèle de croissance de la forme : $\mathrm{B}=\mathrm{A}$. Ip ${ }^{a} \cdot[1-\exp (-\mathrm{k} \cdot \mathrm{t})]^{\mathrm{m}}$ (B : masse ligneuse totale du peuplement; Ip : indice de productivité défini à partir de la hauteur dominante; $t$ : âge du peuplement) permet d'expliquer 97,5 p. 100 de la variance de $B$.

Les courbes de croissance ainsi définies décrivent la production utile en masse ligneuse. La production courante est maximale à 5 ans, juste à la suite de la fermeture du couvert. La production moyenne culmine à 10 ans, et vaut alors 6 tonnes de matière sèche par hectare et par an, pour un peuplement moyen de la région.

Mots clefs : Taillis, Robinia pseudacacia, biomasse, production, régression, modèle de croissance, indice de productivité.

\section{Introduction}

Cette étude s'inscrit dans le cadre d'une évaluation des potentialités de production en biomasse des différentes essences rencontrées dans les taillis existants. Notre contribution concerne l'espèce robinier (Robinia pseudacacia L.), légumineuse subspontanée en Europe, originaire du Centre-Est des Etats-Unis, qui a été présentée dans diverses monographies (VAulot, 1914; Fowells, 1965; Keresztesi, 1983). Cette espèce possède des caractéristiques intéressantes répondant à certains problèmes spécifiques du taillis : sa symbiose avec un rhizobium fixateur d'azote favet.

Adresse actuelle: INRA, Station d'Agronomie, Domaine Saint-Paul, B.P. 91, F 84/40 Mont- 
(Moiroud \& Capellano, 1982 ; Hirschfeld et al., 1984) peut éviter les baisses de fertilité azotée liées à l'exportation fréquente de petit bois (Bouchov et al., 1985); elle possède aussi la faculté de drageonner puissamment, et laisse donc espérer un certain renouvellement de l'ensouchement au cours des rotations successives, ensouchement qui dans d'autres taillis tend à s'affablir, aussi bien en nombre de souches par unité de surface, qu'en efficacité des souches restantes (Crowther \& Evans, 1984).

Dans la région forestière "Val-de-Loire », où nous avons travaillé, les peuplements de robinier se présentent sous forme de taillis brise-vent dans le fond de vallées (bosquets de l'ordre du demi-hectare), et sous forme de taillis plus étendus sur les terrasses plus anciennes, se mélangeant alors avec d'autres espèces. Ces peuplements font le plus souvent partie de la forêt privée paysanne. Les meilleurs bois servent à fabriquer divers piquets et tuteurs, et le reste est utilisé pour le chauffage. Pour répondre à ces utilisations, le robinier est traité en taillis, avec une durée de rotation variant entre 15 et 25 ans. De plus en plus, l'ensemble de la matière ligneuse de ces taillis est déchiqueté en "plaquettes» qui servent de combustible fluide pour le chauffage de lotissements (AuCLaIr et al., 1985).

Notre but est ici de préciser les lois d'évolution de la masse ligneuse sur pied tout au long d'une rotation, pour le taillis pur de robinier, dans les milieux de la région. La masse sur pied est à tout moment la masse récoltable, sa variation est la production utile, qui constitue un bilan entre la production en biomasse et la mortalité. Nous ne ferons pas la distinction entre biomasse et nécromasse et parlerons globalement de "masse ligneuse totale sur pied ». La séparation entre biomasse et nécromasse, tout en étant intéressante pour le biologiste (Duvigneaud, 1971 ; Kestemont, 1971 ; Herbert, 1982), est fort délicate à faire sur le robinier, car les organes vivants et les organes morts sont difficilement séparables.

\section{Matériel}

\subsection{La région}

Le climat de la région est de type océanique à légère tendance continentale (pluviométrie moyenne : 600 à $700 \mathrm{~mm}$ par an; température moyenne de juillet : 18 à $21^{\circ} \mathrm{C}$; température moyenne de janvier : 2 à $4^{\circ} \mathrm{C}$ ).

Les peuplements étudiés se trouvent sur la séquence d'alluvions de la Loire qui constituent un système de terrasses emboîtées d'âges différents. Les sols actuels se développent sur la couverture hydroéolienne du Würm, de nature sableuse, quelquefois très légèrement limoneuse, et d'épaisseur variable. Des paléosols sont en position de substratum (Horemans, 1961 ; PAGÈs, 1985).

\subsection{Les taillis}

Les taillis observés sont âgés de 1 à 25 ans, ils ont subi un nombre variable et inconnu de rotations. 


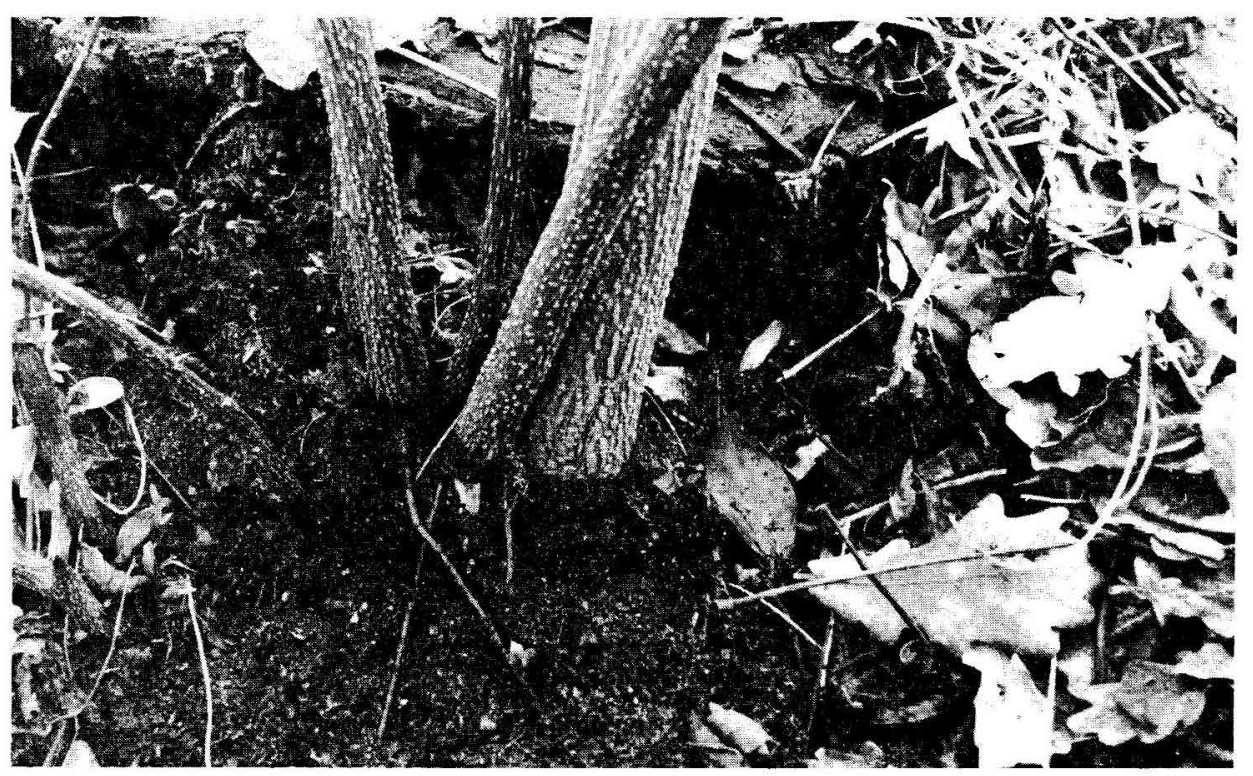

Рното 1

Base des rejets de I an issus d'un même bouton épicormique.

Base of first-year shoots, coming from the same epicormic bud.

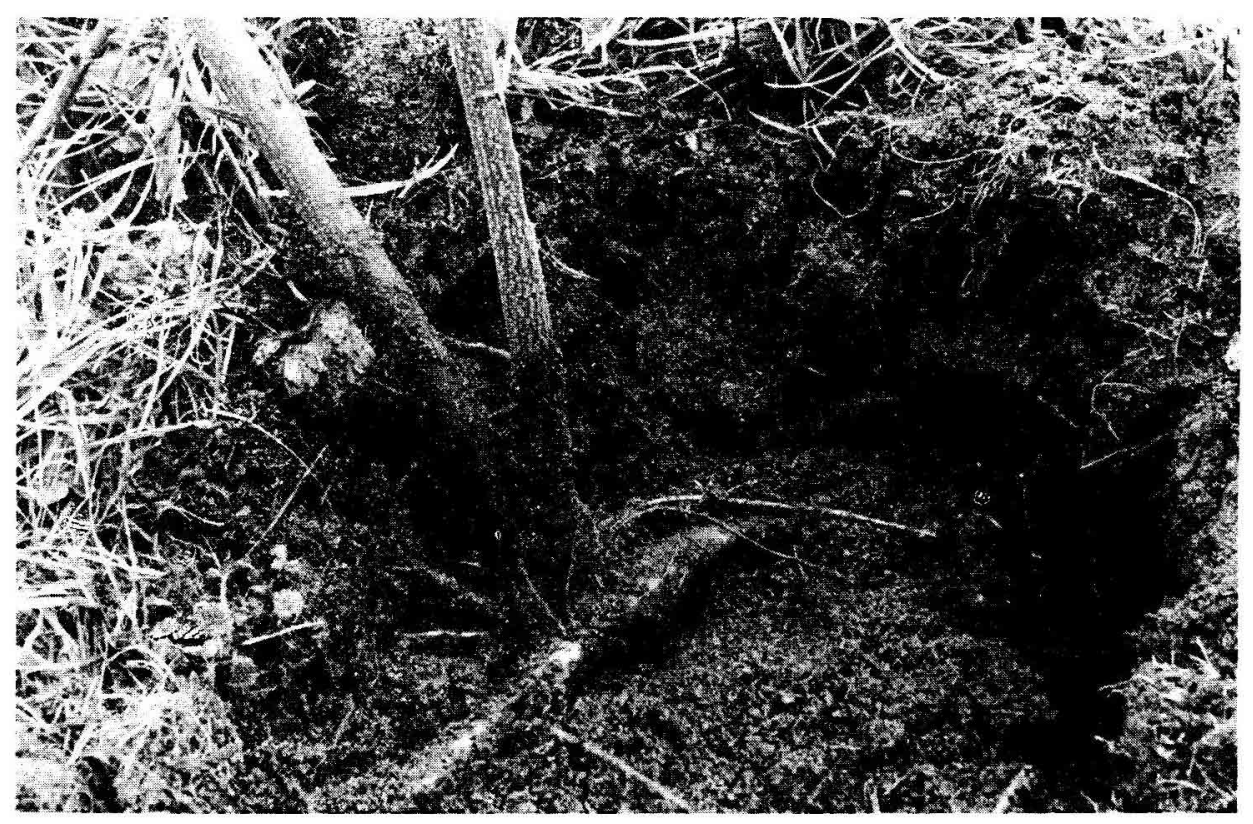

Рното 2

Drageons.

Root suckers. 
Dans le jeune taillis, les rejets ne sont pas régulièrement dispersés sur la surface du peuplement, mais regroupés sur deux niveaux :

- au niveau du "bouton épicormique" (JAcoBs, 1955; RIEDACKER, 1973), amas de bourgeons qui donne naissance à un groupe de rejets fusionnés à leur base (photo 1);

- au niveau de la cépée (photo 3) : les boutons épicormiques sont eux-mêmes regroupés autour des étocs de la rotation précédente.

Sur cette essence, on trouve aussi des brins plus ou moins isolés, issus de l'appareil souterrain : ce sont des drageons (photo 2). Au fur et à mesure du vieillissement du taillis, une sélection s'opère parmi les brins, d'abord au niveau du bouton, puis de la cépée. Le peuplement évolue ainsi vers une structure où le regroupement des brins est de moins en moins visible et les brins isolés proportionnellement plus abondants (photo 4).

L'évolution de la densité en fonction de l’âge est représentée sur la figure 1.

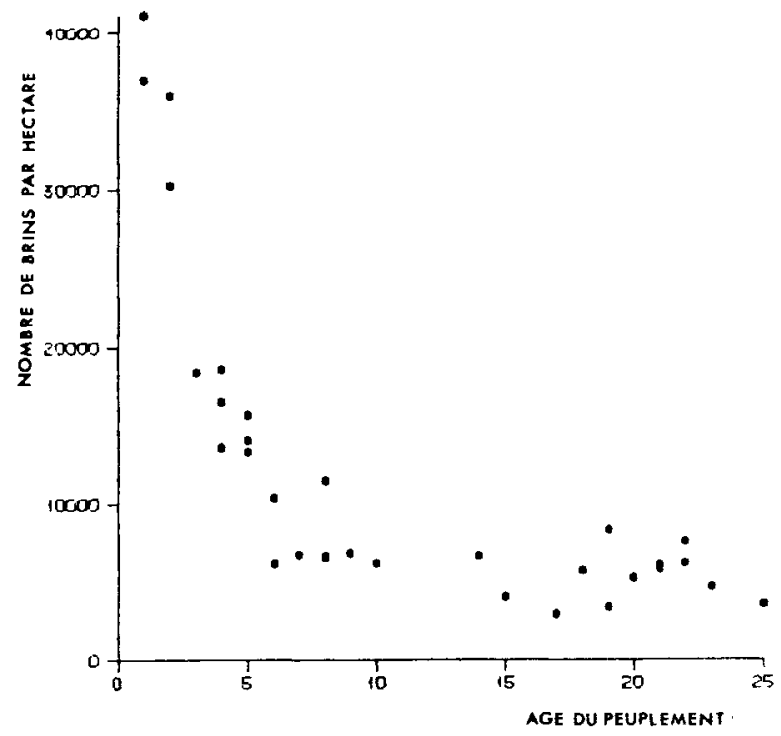

Fik. 1

Evolution de la densité des peuplements en fonction de leur âge.

Evolution of stand density (number of shoots por hectare) with age.

Chaque point représente un peuplement.

Each dot represents one stand.

La fonction de distribution des effectifs de brins par classes de circonférence (telle que celle de la figure 2 , relative à un peuplement de 8 ans) présente un mode nettement décalé vers la gauche. Cette asymétrie est particulièrement accusée dans 
les jeunes peuplements, elle tend à s'atténuer au fur et à mesure que le taillis vieillit. Nous avons également noté le caractère inéquienne de ces taillis (PAGÈs, 1985), montrant que le peuplement continue de rejeter plusieurs années après la coupe. Ce sont essentiellement des drageons qui apparaissent alors, et tendent à occuper les plus grands espaces entre les cépées.

La fermeture du couvert et la fructification des peuplements s'opèrent vers l'âge de 4 ans.

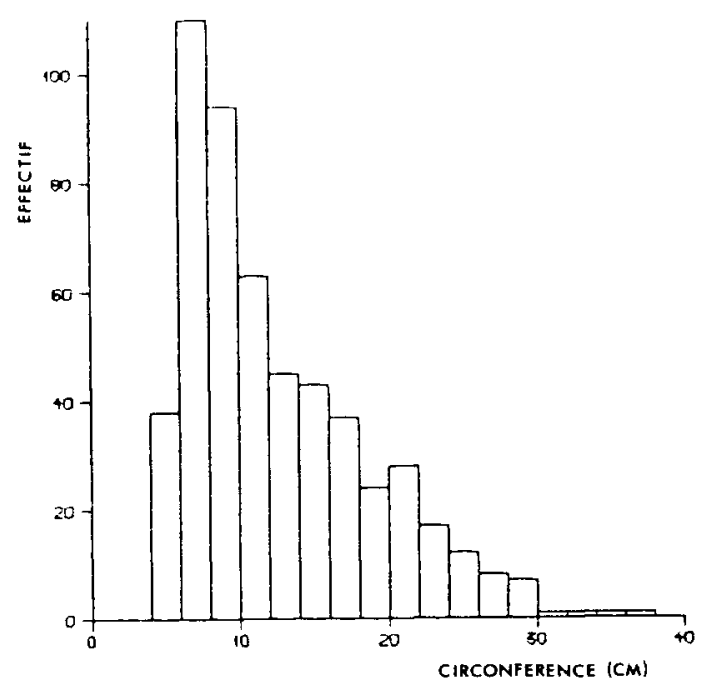

Fig. 2

Histogramme de distribution des circonférences à $1,30 \mathrm{~m}$ dans un peuplement de 8 ans. Histogram showing the distribution of girth at breast-height in one 8-year-old stand.

\section{Méthodes}

Nous avons reconstitué l'évolution de la masse sur pied au long d'une rotation en la mesurant sur un ensemble de peuplements âgés de 1 à 25 ans. Pour ce faire, nous avons opéré de façon classique en procédant à un échantillonnage en deux étapes :

- lot de tiges individuelles sur lesquelles on fait les mesures de masse ligneuse totale, que l'on relic à des variables facilement mesurables sur les tiges sur pied, par des "tarifs";

- lot de placettes-échantillons sur lesquelles on fait un inventaire des variables « entrées du tarif» sur tous les arbres individuels.

Cette démarche est donc suivie d'une double extrapolation : on estime par le tarif la grandeur biomasse sur les placettes inventoriées, puis sur l'ensemble des peuplements étudiés. 


\subsection{Echantillon des placettes inventoriées}

Un ensemble de peuplements de taillis de robinier nous a été indiqué par le Centre régional de la propriété forestière (région Centre). A l'intérieur de chacun d'eux, nous avons installé des placettes circulaires, de centres tirés au hasard, répondant à certaines contraintes : pureté (à plus de 95 p. $100 \mathrm{en}$ nombre de tiges), taillis non dominé, éloignement suffisant de toute discontinuité marquée.

La taille de ces placettes a été déterminée à l'aide d'une méthode empirique (PAGÈs, 1985) de façon à régulariser la microstructure existant dans un peuplement (Duplat \& Perrotte, 1983). Leur surface varie de $100 \mathrm{~m}^{2}$ (pour les plus jeunes peuplements) à $400 \mathrm{~m}^{2}$ (pour les plus âgés).

Finalement, nous avons retenu 31 placettes de ce type, dont les âges ont été le plus régulièrement possible répartis entre 1 et 25 ans.

\subsection{Construction des tarifs}

Les tarifs ont été établis sur l'individu brin. Pour différentes espèces, dont le robinier, il ressort que la prise en compte du facteur cépée n'apporte pas de précision supplémentaire dans le tarif (Auclair \& Metayer, 1980; Francis, 1984; Pagès, 1985). Les variables explicatives du tarif ne seront done que des mesures relatives au brin.

L'outil statistique utilisé est la régression linéaire pondérée.

L'échantillon des brins pesés, constitué de 217 tiges, a été stratifié :

- en fonction de l'âge : nous n'avons pu faire des prélèvements de brins que sur 8 peuplements différents, âgés de 1 an, 2 ans, 3 ans, 4 ans, 6 ans, 8 ans, 14 ans, 21 ans;

- en fonction de la circonférence : pour chaque classe de circonférence, nous avons pris un effectif proportionnel au produit (effectif de la classe de circonférence dans la population "ensemble des placettes») $\times$ (variance a priori de la biomasse de cette classe de circonférence). D'après des études précédentes (Bouchon, 1974; Auclair \& Metayer, 1980 ; Herhert, 1982), la variance de la biomasse dans chaque classe peut être supposée proportionnelle à (circonférence ${ }^{4}$ ). Cette stratégie permet d'obtenir, en utilisant la régression pondérée par l'inverse de la variance, un « centrage pondéré » de l'échantillon par rapport à la population (PERrotTe, 1976).

Sur ces brins-échantillons, les masses ligneuses totales ont été estimées suivant le protocole défini par Auclair \& Metayer, 1980; il s'agit de masses séchées à $105^{\circ} \mathrm{C}$ jusqu'à poids constant.

\subsection{Le modèle de croissance}

La reconstitution des courbes d'évolution de la masse sur pied des peuplements a été obtenue en ajustant des modèles de croissance déterministes aux 31 données (une par placette inventoriée). 
C'est ici la technique de régression non linéaire qui a été utilisée. L'estimation des paramètres est faite par réitération, suivant le critère du maximum de vraisemblance, la distribution des erreurs étant supposée gaussienne (BOUVIER et al., 1985; HuET, 1985). Les tests d'hypothèses (modèles contraints, sous-modèles) utilisent le rapport de vraisemblance comparé à un $\chi^{2}$ (MESSEAN, 1982).

N.B. : Pour tous les tests d'hypothèses, le seuil statistique ne sera pas rappelé et : pris égal à 5 p. 100.

\section{Résultats}

\subsection{Les tarifs}

Pour construire les tarifs, nous avons traité séparément le cas des brins de plus de $1,30 \mathrm{~m}$ de hauteur, et celui des brins de moins de $1,30 \mathrm{~m}$.

En ce qui concerne les premiers, l'échantillon comprend 182 brins. Nous avons testé l'introduction des 3 variables explicatives : c, circonférence à $1,30 \mathrm{~m}$ du brin ; $\mathrm{h}$, hauteur totale du brin ; HD, hauteur dominante du peuplement d'appartenance du brin, définie comme la moyenne des hauteurs des 3 plus gros brins de la placette. Ces variables ont été introduites sous forme de relations allométriques, du type :

$$
\begin{aligned}
& b=a_{0}+a_{1} \cdot c^{\alpha} \\
& b=a_{0}+a_{1} \cdot c^{\alpha} \cdot h^{\beta} \\
& b=a_{0}+a_{1} \cdot c^{\alpha} \cdot H D^{\beta}
\end{aligned}
$$

Les paramètres non linéaires sont estimés par réitération, en maximisant la vraisemblance. La contrainte de ces paramètres à des valeurs $\alpha_{0}$ et $\beta_{0}$ rend les modèles linéaires pour l'ajustement des coefficients $a_{0}$ et $a_{1}$.

Les différents tarifs obtenus sont présentés sur le tableau 1.

Les brins plus petits que $1,30 \mathrm{~m}$ représentent une masse non négligeable dans les peuplements de 1 an et 2 ans, il convenait donc de faire un tarif particulier, ici établi sur 35 individus. Nous avons obtenu la relation :

$$
b=0,64+0,041 \cdot h^{2,3} \quad(b \text { en } g, h \text { en } d m)
$$

qui explique 78,3 p. 100 de la variance de b.

\subsection{Application aux inventaires}

Ce sont les modèles (3) et (4) qui ont été retenus, car ce sont ceux qui nous ont semblé avoir le meilleur rapport (précision/quantité de travail de mesures). Sur les placettes, nous avons donc réalisé un inventaire exhaustif des circonférences à $1,30 \mathrm{~m}$, et une estimation de la hauteur dominante (moyenne des hauteurs des 3 plus gros brins de la placette). 
BIOMASSE DU TAILLIS DE ROBINIER

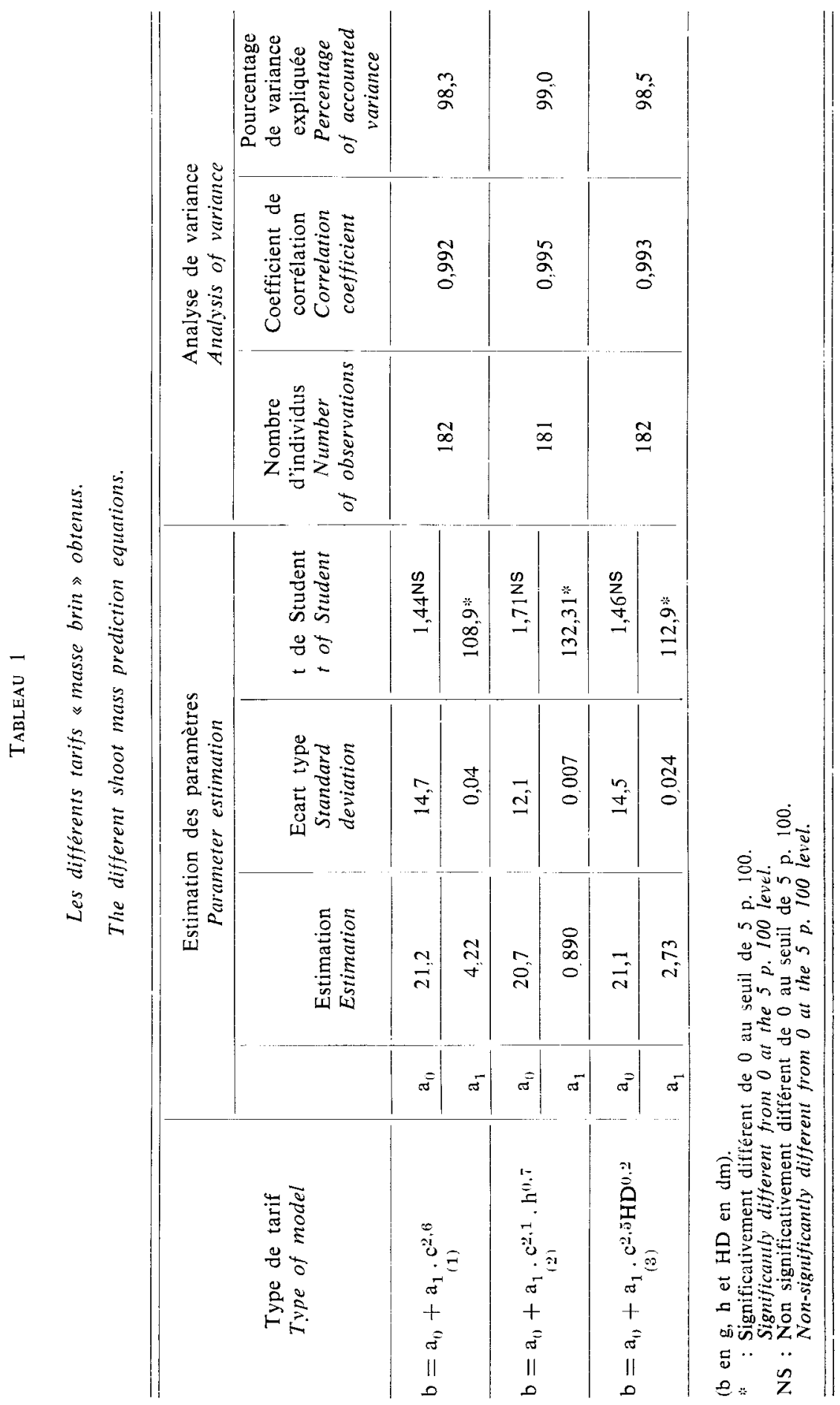


L'application des tarifs aux inventaires nous a permis d'estimer la masse existant sur chaque placette, ainsi que la précision sur l'estimation, selon une formule exposée par Perrotte, 1976. L'erreur relative varie de 2 p. 100 pour les placettes les plus âgées, à 20 p. 100 pour les plus jeunes. C'est essentiellement l'utilisation du tarif (4) qui baisse la précision globale. Les variances d'estimations ainsi obtenues ont été utilisées pour pondérer les régressions ultérieures.

Le nuage des masses ligneuses totales à l'hectare des différentes placettes, en fonction de leur âge, est représenté sur la figure 3 .

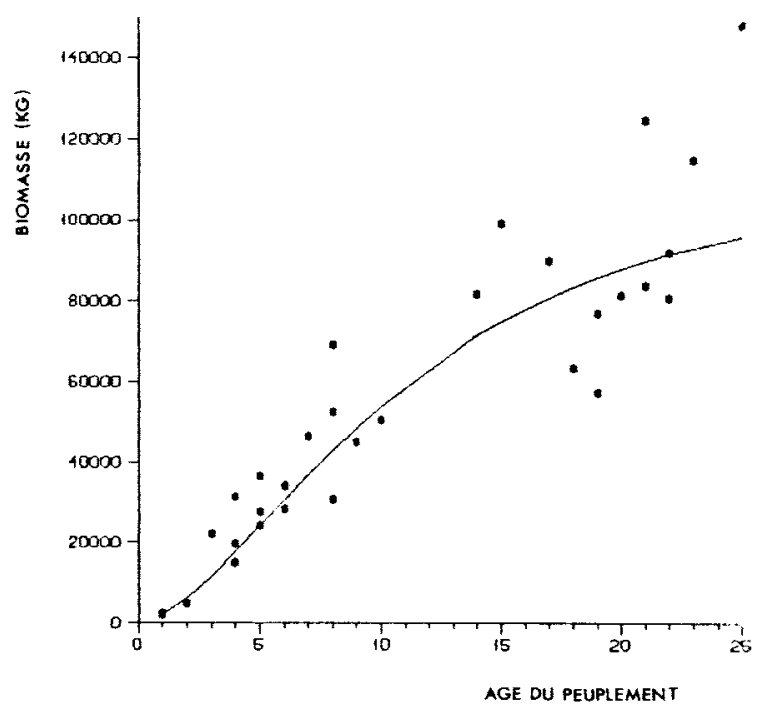

FIG. 3

Relation entre la masse ligneuse totale à lhectare et lâge du peaplement.

Relation between the total woody mass per hectare and the age of the stand.

Les points sont les valeurs observées, ta courbe représente le modèle de croissance moyen (6).

Dots are observed values, the curve represents the mean growth model (6).

\subsection{Modèle d'évolution de la masse sur pied}

De façon à décrire les variations temporelles de masse sur pied, nous avons utilisé un modèle de croissance déterministe : la fonction logistique généralisée. Ce modèle a été formulé et paramétré de manières différentes par divers auteurs (cités par Debouche, 1979 ; Lebreton \& Millier, 1982 ; Jolivet, 1983). Sous forme explicite, il peut s'écrire :

$$
Y=A \cdot\left[1-\exp \left(-k \cdot\left(t-t_{1}\right)\right)\right]^{m}
$$

(Y est la grandeur étudiée, t est le temps, ou l'âge). 
Ce modèle est efficace pour décrire des croissances en biomasse (PiEnaAR \& Turnbull, 1973), des croissances en hauteur (Osumi, 1983). Il englobe d'autres modèles intéressants : logistique, Gompertz, Mitscherlich, en les généralisant.

A partir du modèle $(5)$, nous avons testé l'hypothèse $t_{0}=0$, qui correspond à une contrainte de la fonction à passer par l'origine. Cette hypothèse nous semble plausible, et elle est statistiquement admissible (cf. tableau 2). Cet ajustement nous donne un modèle moyen :

$$
B=102000 .[1-\exp (-0,121 \cdot t)]^{1, \pi}
$$

avec $B$ en $\mathrm{kg}, \mathrm{l}$ en années.

\section{TABleau 2}

Les différents modiles de croissance ajustés.

The different growth models.

\begin{tabular}{|c|c|c|c|}
\hline & $\begin{array}{l}\text { Modèlc } \\
\text { Model }\end{array}$ & $\begin{array}{c}\text { Nombre de degrés } \\
\text { de liberté } \\
\text { Desrees } \\
\text { of freedom }\end{array}$ & $\begin{array}{c}-2 . \log \\
\text { (vraiscmblance) } \\
-2 . \log \\
\text { (likelihood) }\end{array}$ \\
\hline (5) & $\mathrm{B}=123000 \cdot\left[1-\left.\exp (-0.058 .(\mathrm{t}-0807))\right|^{1,1}\right.$ & 27 & 671,1 \\
\hline (6) & $B=102000 .[1-\exp (-0,121 . t)]^{1.71}$ & 28 & 671,7 \\
\hline (8) & $B=I p^{1.51} \cdot 95600 \cdot[1-\exp (-0,174 . t)]^{2.36}$ & 27 & 6283 \\
\hline
\end{tabular}

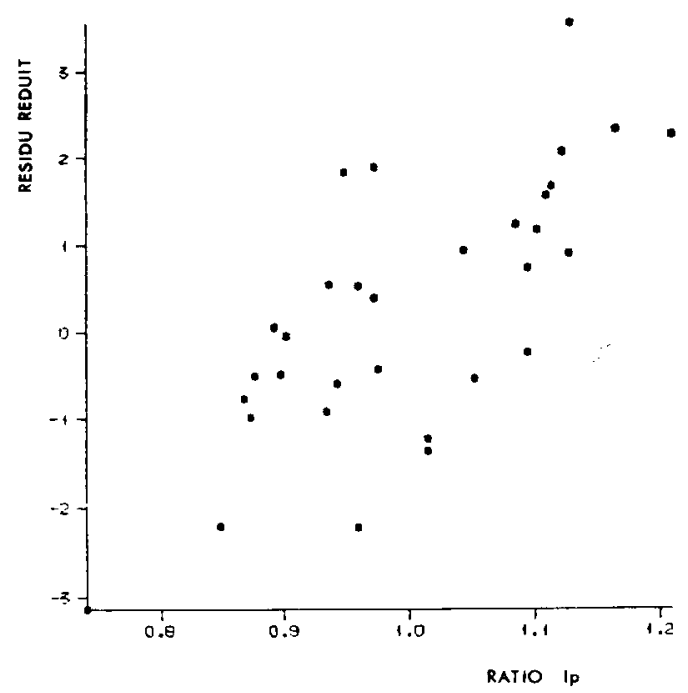

Fici. 4

Graphe des résidus du modèle moyen (6) en fonction de lindice if.

Plot of the mean model (6) residuals versus the Ip index. 
Les résidus de ce modèle moyen grandissent avec la hauteur dominante des peuplements, aussi nous nous sommes intéressé à la variation temporelle de celle-ci. Pour décrire son évolution moyenne, nous avons retenu la relation :

$$
H D=164 .[1-\exp (-0,150 . t)]
$$

avec HD en dm, $t$ en années, après avoir constaté que le modèle plus général du type (5) nous donnait des valeurs de $m$ peu différentes de 1 , et de $t_{0}$ peu différentes de 0 . Nous nous sommes ensuite intéressé au rapport :

$$
I p=\frac{\text { HD (observée) }}{\text { hd moyen [estimée par le modèle (7)] }} \text { (Ip indice de productivité) }
$$

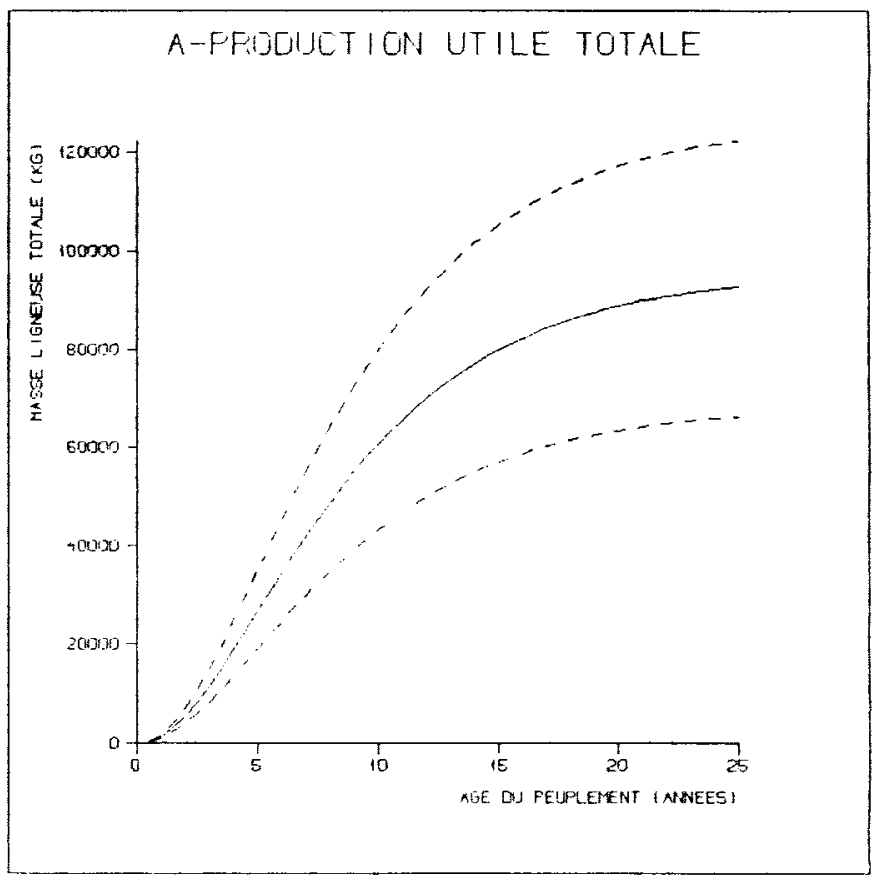

Fig. 5

Courbes de production utile: $A$ - totale, $B$ - courante, $C$ - moyenne. Production curves: $A$ - total, B - current, $C$ - mean.

La courbe en trait plein correspond à la moyenne de la population (Ip $=1)$. Les courbes en tireté correspondent aux extrêmes de la population ( $\mathrm{Ip}=0,8 ; \mathrm{Ip}=1,2$ ). Les chiffres sont rapportés à l'hectare.

The solid line represents the population mean $(I p=1)$. The dashed-lines represent the lowest and highest values of the population $\left(I p=0.8 ; I_{p}=1.2\right)$. The given values are computed on a per hectare basis. 
B-PRODUCTION UTILE COURANTE

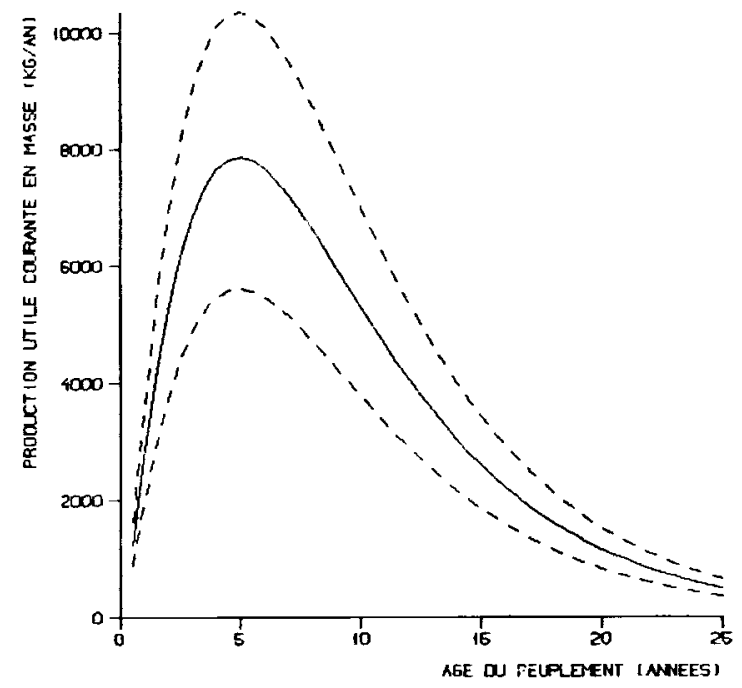

C-PRODUCTION UTILE MOYENNE

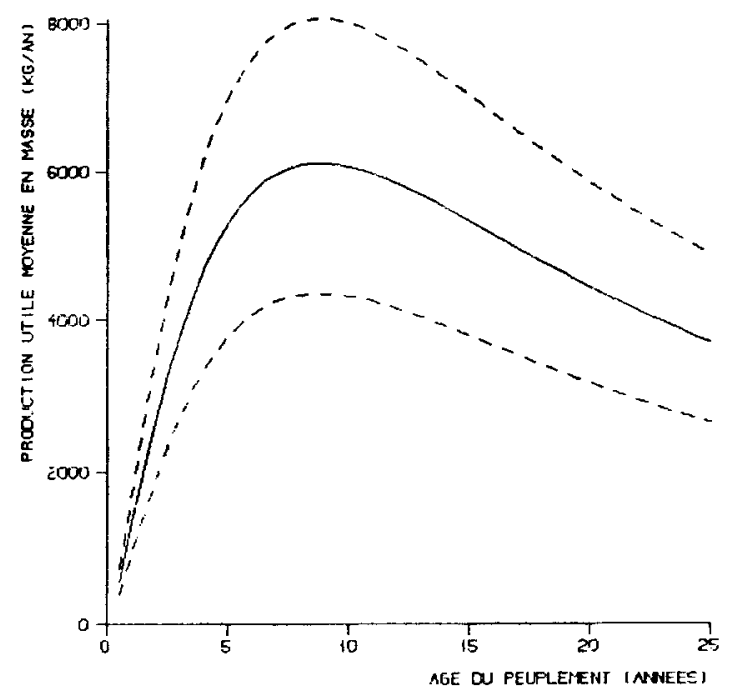


Cet indice traduit l'allongement relatif d'un peuplement donné par rapport à l'allongement moyen des peuplements de la population, au même âge. La figure 4 montre la relation entre les résidus du modèle (6) et le rapport Ip. Cette visualisation nous a incité à tester le nouveau modèle :

$$
B=A \cdot I p^{\mu} \cdot[1-\exp (-k \cdot t)]^{m} \quad(c f . \text { tableau 2) }
$$

La date d'inflexion est $5 \pm 1$ ans (intervalle de confiance à 5 p. 100). Cette relation nous permet de calculer la masse sur pied, production courante utile et production moyenne utile, en fonction de l'âge (cf. figure 5).

\section{Discussion}

Le modèle proposé ici pour décrire la production utile en masse ligneuse ne doit pas être considéré comme définitif, même s'il nous a déjà semblé intéressant de le présenter sous cette forme. Notons tout d'abord qu'il n'a été établi qu'à partir de 31 observations instantanées de peuplements. Le tarif biomasse, qui est à l'origine de cette construction, n'a été réalisé, à cause de contraintes pratiques, qu'à partir de 8 peuplements différents. Il est donc possible que cet échantillon de brins pesés ne reflète pas toute la variabilité existant dans la population étudiée. Cependant, il faut remarquer l'étroitesse de la liaison entre la masse ligneuse du brin et les variables prédictives choisies, puisque le tarif explique 98,5 p. 100 de la variance de cette masse. D'autre part, nous avons bâti un indice de productivité très simple défini à partir d'une courbe moyenne de hauteur dominante. Il faut souligner que cette façon de procéder est quelque peu dangereuse, car elle suppose que l'échantillon de placettes ayant servi à la construire soit bien équilibré vis-à-vis des différentes fertilités, dans chaque classe d'âge, de façon à ce que cette courbe de référence soit bien moyenne. Notons que ce premier modèle, aussi simple soit-il, permet d'expliquer 97,5 p. 100 de la variance de la masse ligneuse totale.

On peut également s'interroger sur le type de modèle utilisé. Chaque modèle présente un certain nombre de contraintes, en liaison avec son nombre de paramètres. Ici, nous avons délibérément choisi un modèle qui présente une asymptote horizontale. Cette hypothèse nous semble parfaitement acceptable dans la mesure où l'on s'intéresse à l'évolution de la masse sur pied, qui «plafonne » naturellement. DuvigneAud, 1974, explique cependant que "l'équilibre" atteint au bout d'un certain temps est soumis à des fluctuations, explicables par les variations climatiques, le vieillissement d'une population, l'accumulation dans le milieu de métabolites toxiques, ... Ce modèle est par contre tout à fait flexible quant à la position du point d'inflexion.

Les données de production en masse totale concernant les taillis existants sont souvent très ponctuelles. Le modèle proposé permet de constater que la production moyenne utile maximale serait obtenue pour une exploitation dès 10 ans. Elle est alors d'environ 6 tonnes de matière sèche par hectare et par an, pour un peuplement moyen de la région. La production courante utile culmine vers 5 ans. On peut mettre cette date en relation avec l'époque de fermeture du couvert, comme l'ont observé divers auteurs sur d'autres essences ou d'autres types de peuplements (FALKEnHagen, 
1980; SHen et al., 1984). Notons aussi que les débuts de la fructification du taillis de robinier interviennent également vers cet âge, et pourraient accentuer le phénomène d'inflexion de la croissance ligneuse.

Pour compléter ce premier modèle de croissance, il nous semble important d'élargir l'échantillonnage, aussi bien en pratiquant des analyses de tiges, qu'en pesant d'autres brins et en inventoriant d'autres placettes. Le complément à notre échantillon doit se faire en soignant la stratification vis-à-vis des types de milieux. La poursuite de cette étude passe donc par la réalisation d'une typologie des stations à robinier dans la région. D'autre part, il serait intéressant d'étudier comment le modèle peut être réitéré d'une rotation à la suivante. Pour ce faire, les données dendrométriques peuvent être complémentées par une enquête visant à connaître le passé sylvicole des différents peuplements (nombre de rotations, durée des rotations).

Reģu le 19 février 1986.

Accepté le 22 mai 1986.

\section{Remerciements}

Nous tenons à remercier les propriétaires forestiers de la région pour leur accueil, ainsi que le Centre régional de la propriété forestière, et tout particulièrement M. Formery, pour son aide lors de la prospection.

\section{Summary \\ Biomass growth curves : the example of Black Locust in the French «Val-de-Loire » region.}

Our aim is to study the biomass production potentialities of traditional coppice, for various species, sites and managements. This contribution deals with Black Locust (Robinia pseudacacia L.) in the "Val-de-Loire» region. This species, widely introduced in the region, is interesting in coppice management, because of its nitrogen-fixing and rootsuckering ability. Our measures concern the evolution of standing woody mass during the classical coppice-cycle (25 years), without separating living and dead parts of trees.

The studied coppice stands are 1 to 25 years old, and have been cut a variable unknown number of times. They have grown on alluvial sandy soils, under oceanic climate.

31 circular sample plots from 100 to $400 \mathrm{~m}^{2}$ have been surveyed in pure coppice. In each plot, girth at breast-height of every shoot, and dominant height of the plot have been measured. In young stands, height of small shoots - smaller than $1.30 \mathrm{~m}$ - has also been measured. From a sample of 217 shoots of various ages, mass prediction equations were built. Two equations were applied on the different inventories :

$$
\text { (1) } b=a+a_{1} \cdot c^{\alpha} \cdot H D^{*}
$$

(b : total woody mass; $c$ : girth at breast height; HD : dominant height of the stand), for shoots higher than $1.30 \mathrm{~m}$, and :

$$
\text { (2) } b=a_{n}+a_{1} \cdot h a
$$

(h: height of the shoot) for shoots smaller than $1.30 \mathrm{~m}$.

Different growth curves have been fitted to the computed values of total standing woody mass. The ones finally adopted are :

$$
\text { (3) } B=A \cdot I p^{a} \cdot[1-\exp (-k \cdot t)]^{m}
$$


( $\mathrm{B}=$ standing woody mass, $\mathrm{t}=$ age of the stand), were $\mathrm{Ip}$ is delined as a ratio of the observed dominant height to the mean dominant height, computed with a similar model.

These growth curves describe the variations of potential crop with age and fertility. They show that maximum current annual increment is reached at the age of 5 years, when the stand has just closed. The maximum mean annual increment is reached at the age of 10 years, and at this time, the crop could yield 6 metric tons per hectare and per year, for a mean stand of this region.

Aditional measurements are suggested to improve the accuracy of the Inodel, and to help describe the yield along successive coppice-cycles.

Key words : Coppice, Robinia pseudacacia, biomass, production, regression, growth model, site index.

\section{Références bibliographiques}

Auclair D., Metayer S., 1980. Méthodologie de l'évaluation de la biomasse aérienne sur pied et de la production en biomasse des taillis. Acta Oecologica, Oecol. Applic.. 1 (4), 357-377.

Auclalr D., Jorez. J.P., Piermont L., 1985. Variabilité des qualités énergétiques de plaquettes de bouleau en Sologne. Rev. For. Fr., XXXVII (5), 389-397.

Bouchon J., 1974. Les tarils de cubage. Institut national de la recherche agronomique, Centre national de recherches forestières. Ecole nationale du génie rural, des eaux et des forêts, Nancy, $57 \mathrm{p}$.

Bouchon J., Nys C., Ranger J., 1985. Cubage, biomasse et minéralomasse : comparaison de trois taillis simples des Ardennes primaires. Acta Oecologica, Oecol. plant., 6 (1), $53-72$.

Bouvier A., Gelis F., Huet S., Messean A., Neveu P., 1985. Manuel d'utilisation de CS-NL. Institut national de la recherche agronomique, laboratoire de Biométrie, Jouy-en-Josas, $180 \mathrm{p}$.

Crowthli:R R.E., Evans J., 1984. Coppice. Forestry commission leaflet, 83, 23 p.

Debouche C., 1979. Présentation coordonnée de modèles de croissance. Rev. Stat. Appl., $27(4), 5-22$.

Duplat P., Perrotte G., 1983. Inventaire et estimation de l'accroissement des peuplements forestiers. Office national des forêts, section technique, $432 \mathrm{p}$.

Duvigneaud P., 1971. Concepts sur la productivité primaire des écosystèmes forestiers. UNESCO, 1971. Productivité des écosystèmes forestiers, Actes du colloque de Bruxelles. 1969. Ecologie et conservation, 4, 111-140.

Duvigneaud P., 1974. La synthèse écologique. Doin, Paris, 296 p.

Falkenhagen E.R., 1980. The law of Eichhörn : use, extension and applicability in South Africa. South African Forestry Journal, 114, 7-12.

Fowells H.A., 1965. Silvics of forest trees of the United States. Division of timber management research. U.S.D.A. forest service, agriculture handbook, 271, 761 p.

FrancIS J.K., 1984. Biomass accumulation by single - and multiple - stemmed young Sycamore. Forest Science, 30 (2), 372-374.

Herbert I., 1982. Evolution de la biomasse et de la nécromasse dans un jeune taillis de châtaigniers de 12 ans. D.E.A. d'écologie végétale. Université de Paris-Sud, 31 p.

Hirschfeld J.R., Finn J.T., PATTLRSON W.A., 1983. Effects of Robinia pseadoacacia on leaf litter decomposition and nitrogen mineralization in a northern hardwood stand. Can. J. For. Res., 14, 201-205.

Horemans P., 1961. Contribution à l'étude pédologique des terrasses alluviales de la Loire moyenne. Thèse de $3^{\text {" }}$ cycle de l'Université de Paris-Sud.

HUET S., 1985. Maximum likelihood and least squares estimators for a non-linear model with heterogeneous variances. Statistics (à paraître). 
JacoBs M.R., 1955. Growth habits of Eucalyts. Government printer, Canberra (Australia), $261 \mathrm{p}$.

Jolivet F., 1983. Introduction aux modèles mathématiques en biologie. Actualités scientifiques et agronomiques de I'INRA, 11. Masson, Paris, $151 \mathrm{p}$.

Kereszresi B., 1983. The Black Locust. Unasylva, 32 (127), 23-33.

Kestemont P., 1971. Productivité primaire des taillis simples et concept de nécromasse. UNESCO, 1971. Productivité des écosystèmes forestiers, Actes du colloque de Bruxelles, 1969. Ecologie et conservation, 4, 271-279.

Lebreton J.D., Millier C., 1982. Modèles dynamiques déterministes en biologie. Masson, Paris, $207 \mathrm{p}$.

Messean A., 1982. Régions de confiance dans le modèle non-linéairc. Journal de la Société de Statistique de Paris, 134-143.

Moiroud A., Capeli.nno A., 1982. Le robinier, Robinia pseudoacacia L., une espèce fixatrice d'azote intéressante? Ann. Sci. For., 39 (4), 407-418.

Osum S., 1983. Applicabilité de la fonction de Richards à l'analyse de croissance de l'arbre, IUFRO, groupe sectoriel $\$ 4.01-00$; mesures, croissance et production. Les colloques de I'INRA, 19, 77-86.

PAGÈs L., 1985. Les taillis de robinier du Val-de-Loire : croissance, biomasse, régénération. Thèse de doctorat de l'Université de Paris-Sud, $74 \mathrm{p}$.

PERROTIE G., 1976. La régression linéaire multiple pondérée en vue de l'application aux calculs des tarifs de cubage et des tables de production. Ecole nationale du génie rural, des eaux et des forêts, Nancy, $14 \mathrm{p}$.

Pienatr L.V., Turnbuli K.J., 1973. The Chapman-Richards generalization of Von Bertalanffy's growth model for basal area growth and yield in even-aged stands. Forest Science, $19(1), 2-22$.

Riedacker A., 1973. Les taillis d'eucalyptus an Maroc. Annales de la Recherche Forestière au Maroc, 13, 157-349.

SHEN S.Y., VyAS A.D., JonES P.C., 1984. Economic analysis of short and ultra-short rotation forestry. Resources and Conservation, 10, 255-270.

Vaulot G., 1914. Le robinier faux-acacia... Histoire, description, culture, propriétés et utilisations. Baillière, Paris, 264 p. 